\title{
Bewährtes und Neues in der diagnostischen/operativen Phlebologie
}

L. Rafi-Reichrath ${ }^{1}$

D. Stenger ${ }^{2}$

S. Nestoris ${ }^{1}$

E. Dippel ${ }^{1}$

\section{Approved and New Methods in Diagnostic/Surgical Phlebology}

\section{Zusammenfassung}

Die Dermatologische Klinik in Lemgo ist schwerpunktmäßig eine operativ ausgerichtete Klinik. Bereits seit 1987 unter der Leitung von Prof. Dr. Roland Müller waren die Schwerpunkte im Bereich der Tumorchirurgie und Phlebochirurgie. Auch nach der Neubesetzung durch PD Dr. Edgar Dippel im Jahr 2004 wurden die Schwerpunkte fortgeführt und ausgebaut. In unserer Klinik liegen idealerweise Diagnostik, operative Planung und Ausführung in einer Hand. Die Bedeutung der Phlebologie wurde durch zahlreiche epidemiologische Studien belegt. So entwickelten sich nicht zuletzt durch die steigende Prävalenz der primären Varikose und die steigende Anzahl an daraus resultierenden Komplikationen (chronisches Ödem, trophische Hautveränderungen, Ulcus cruris, tiefe Leitveneninsuffizienz, Varikophlebitis) wichtige Fortschritte bei der phlebologischen Diagnostik z.B. mithilfe der Duplexsonographie. Therapeutische Entwicklungen sind insbesondere bei der chirurgischen Vorgehensweise der Rezidivvarikose und bei den endoluminalen Laserverfahren zu finden.

\section{Abstract}

The former leadership of Prof. Dr. Roland Müller gave the clinic of dermatology in Lemgo a dermatosurgical direction. The main therapeutic spectrum is tumor and phlebosurgery. The aim of the current leadership by PD Dr. Edgar Dippel is to continue and to improve this process for example using new apparative phlebological measuring methods and by the use of more differentiated treatment concepts in the phlebosurgical field. In the past few years new interventional methods were taken on, like foam sclerotherapy and endoluminal lasertherapy.

Many recent epidemiologic studies has shown that venous diseases are among the most common diseases in Germany. Venous disorder is common in every age. However it is very important to treat venous diseases at an early stage to avoid complications like oedema, leg ulcer.

\section{Diagnostik}

Primär stellt die exakte und gründliche Diagnostik die Grundlage jeder phlebologischen Therapie dar. Dies gilt für invasive und nicht-invasive Verfahren.

Die ausführliche Anamneseerhebung (Eigen-, Familien-, Berufs-, Medikamenten- und Schmerzanamnese) und die genaue klinische Untersuchung (Inspektion, Palpation) geben häufig Hinwei- se für das Vorliegen einer Venenerkrankung. Es gibt auch eine Reihe von Funktionstests (z.B. Schwartz-Test, TrendelenburgTest, Perthes-Test), die heute nur noch eine untergeordnete Rolle spielen. Für invasive Eingriffe ist die alleinige Aussagefähigkeit dieser Tests meistens zu ungenau.

Institutsangaben

${ }^{1}$ Dermatologische Klinik des Klinikum Lippe, akademisches Lehrkrankenhaus der medizinischen Hochschule Hannover (Chefarzt: PD Dr. E. Dippel)

${ }^{2}$ Arztpraxis, Dermatologie und Phlebologie, Saarlouis

Korrespondenzadresse

Priv.-Doz. Dr. med. Edgar Dippel • Dermatologische Klinik, Klinikum Lippe-Lemgo · Rintelner Straße 85 . 32657 Lemgo · E-mail: edgar.dippel@klinikum-lippe.de 


\section{Apparative Diagnostik}

Das Ziel der apparativen Zusatzuntersuchungen ist es, die klinisch gestellte Diagnose zu bestätigen oder zu differenzieren sowie die insuffizienten Venenabschnitte genau zu identifizieren.

Mit der Ultraschall-Doppler-Sonographie in Kombination mit der klinischen Untersuchung kann man die überwiegende Mehrzahl der phlebologischen Krankheiten einordnen [1]. Die Ultraschall-Dopplersonographie ist meistens vor Durchführung einer konservativen Therapie wie die Kompressionstherapie ausreichend. Vor Durchführung eines operativen Eingriffes sind zusätzliche bildgebende Verfahren indiziert. Hierzu zählt in erster Linie die Duplexsonographie mit oder ohne Farbkodierung. Die Duplexsonographie hat sowohl im epifaszialen als auch im tiefen Venensystem eine Sensitivität von 94-99\% und eine Spezifität von $99-100 \%$. Neben der Refluxdiagnostik ist der Einsatz der Duplexsonographie bei Fragen nach Abstromhindernissen, tiefer Beinvenenthrombose, fortgeleiteter Thrombose bei Thrombophlebitis superficialis indiziert. Die Duplexsonographie ist eine kostengünstige beliebig wiederholbare Untersuchung. Sie ist eine für den Patienten schmerzlose und nicht invasive Untersuchung. Nachteile der Duplexsonographie sind hohe Untersucherabhängigkeit und aufwändige Dokumentation mit langen Untersuchungszeiten.

Die aszendierende Pressphlebographie wurde 1973 von Hach eingeführt und in der phlebologischen Routinediagnostik etabliert [2]. Sie war bis in die 80er-Jahre die Referenzmethode in der phlebologischen Diagnostik. In den letzten Jahren hat sich die Duplexsonographie mehr und mehr etabliert. Die Phlebographie hat damit einen anderen Stellenwert erhalten.

Eine Untersuchung der Arbeitsgruppe von Marshall et al. [3] konnte zeigen, dass Duplexsonographie und Phlebographie eine Sensitivität $\geq 95 \%$ und eine Spezifität $\geq 95 \%$ aufweisen. Allerdings ist die Phlebographie mit höherem Aufwand verbunden als die Duplexsonographie. Zusätzlich stellt die Phlebographie aufgrund der Kontrastmittelverabreichung ein deutlich höheres Risiko für den Patienten dar als die Duplexsonographie. Die Duplexsonographie ist jedoch kostengünstiger als die Phlebographie.

Zusammenfassend kann man sagen, dass die Phlebographie nur in Ausnahmefällen indiziert ist. Sie wird jedoch nach wie vor als weiterführendes bzw. ergänzendes Verfahren eingesetzt.

Die phlebologische Funktionsdiagnostik besteht aus Photoplethysmographie, Venenverschlussplethysmographie und Phlebodynamometrie [4]. Die Photoplethysmographie ist ein Verfahren zur Darstellung der Änderung des Füllungsverhaltens der dermalen Venenplexus unter einem standardisierten Bewegungsprogramm. Sie dient als Screeningtest und hat eine hohe Fehlerquote.

Die Venenverschlussplethysmographie ist ein Verfahren zur Messung der druckabhängigen venösen Kapazität, des venösen Ausstroms und der aktiven Volumenschöpfung. Sie ist wichtig zur Verlaufskontrolle beim postthrombotischen Syndrom.
Die Phlebodynamometrie ist eine blutige Messung des peripheren Venendruckes und dessen Änderung bei Lagerungsproben und unter normierter Belastung. Sie ist der wichtigste präoperative Funktionstest und ermöglicht Funktionsaussagen bei pathologischen Befunden im tiefen Venensystem.

\section{Therapie}

Die therapeutischen Möglichkeiten umfassen konservative Maßnahmen (Kompressionstherapie), Sklerosierungstherapie, operative Verfahren, endoluminale Verfahren und transkutane Lasertherapie.

Gerade in den letzten Jahren haben sich auf dem Gebiet der operativen Phlebologie viele Neuerungen ergeben. Aus unserer Sicht ist der „state of the art“ in der operativen Venenchirurgie folgendermaßen zu definieren: eine sorgfältige, erweiterte Crossektomie kombiniert mit einem invaginierenden Stripping oder StabStipping kombiniert mit der Miniphlebektomie und ergänzt durch die seit wenigen Jahren bekannte Schaumsklerosierung.

Unter der erweiterten Crossektomie verstehen wir Folgendes: suprapubischer Zugang nach Brunner. Freilegung der Crosse und der V. femoralis über einer Länge von $2-3 \mathrm{~cm}$. Niveaugleiche doppelte Ligatur der V. saphena magna mit der V. femoralis mit nichtresorbierbarem Material. Ligatur aller direkt in die V. femoralis einmündender Seitenäste (30\%) mit nichtresorbierbaren Fäden. Koagulation jeglichen freien Endothels (Abb.1).

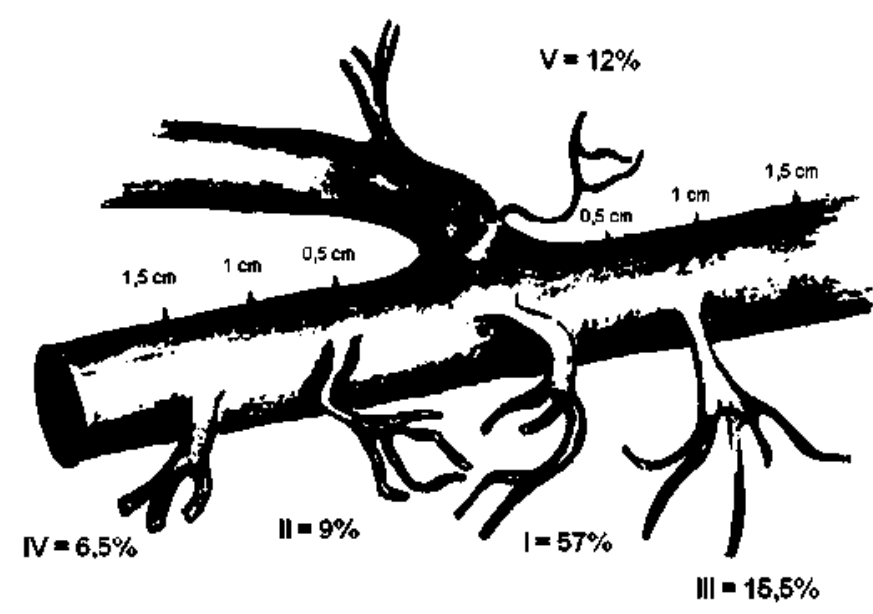

Abb. 1 Schematische Darstellung der erweiterten Crossektomie.

In einer eigenen Untersuchung konnten wir zeigen, dass im Crossebereich in etwa einem Drittel der Fälle direkt in die V. femoralis einmündende Seitenäste vorkommen, die wir mit nichtresorbierbarem Material ligieren. Diese in die V. femoralis einmündenden Seitenäste finden sich in $1,5 \mathrm{~cm}$ proximal bzw. distal des oberen bzw. unteren Pols der V. saphena magna crosse. Am häufigsten sieht man dabei den Typ I (Tab.1).

Zusammenfassend ist hinsichtlich der von uns favorisierten erweiterten Crossektomie Folgendes festzuhalten: Bei $1 \frac{1}{3}$ der Fälle mit Stammvarikose der V. saphena magna finden sich direkt in die V. femoralis einmündende Seitenäste. Die Seitenäste mün- 
Tab. 1 Anzahl der Femoralis-Seitenäste (77/244)

\begin{tabular}{lcc}
\hline & Anzahl (n) & Prozent (\%) \\
\hline Typ I & 44 & 57 \\
\hline Typ II & 7 & 9 \\
\hline Typ III & 12 & 15,5 \\
\hline Typ IV & 5 & 6,5 \\
\hline Typ V & 9 & 12 \\
\hline
\end{tabular}

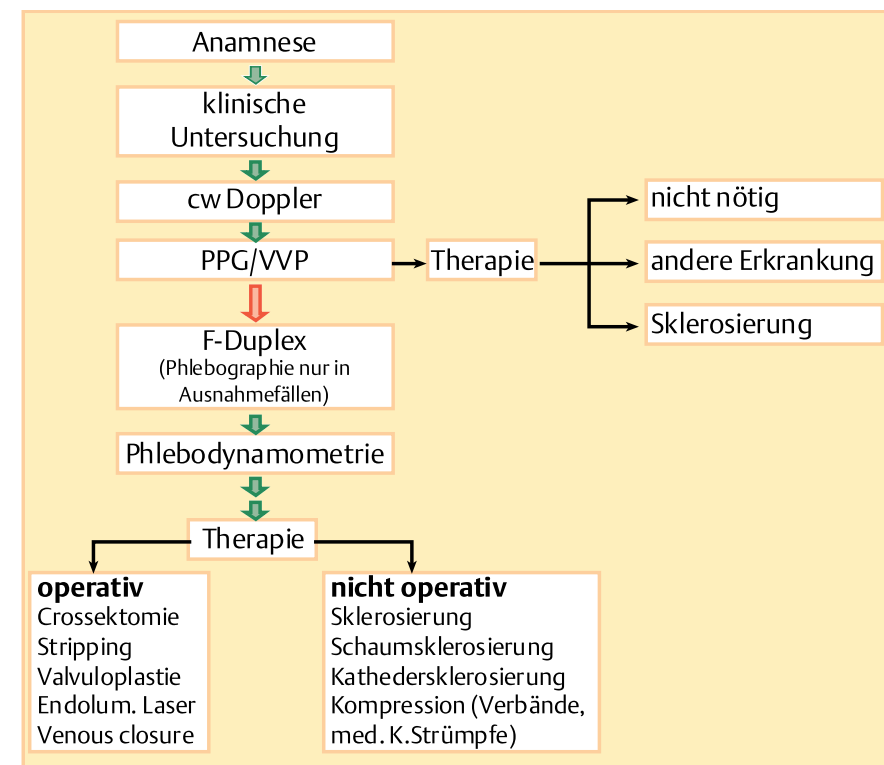

Abb. 2 Algorhythmus der phlebologischen Diagnostik und Therapie.

den fast immer medial. Die längerstreckige Freilegung des Crossebereiches ermöglicht immer eine exakte, niveaugleiche Ligatur der V. saphena magna mit der V. femoralis. Das Risiko der Rezidivvarikose wird durch diese Op-Technik wahrscheinlich reduziert. In unserem Krankengut ist bisher kein erhöhtes Risiko hinsichtlich TVT oder Lungenembolie beobachtet worden.

In unserer Klinik besteht die Möglichkeit den operativen Eingriff sowohl in ITN oder Spinalanästhesie als auch in Tumeszenzanästhesie durchzuführen. Weiterhin favorisieren wir ein invaginierendes Stripping bzw. Stab-Stripping und beschränken uns auf ein partielles Stripping bis zum distalen Insuffizienzpunkt. Hierzu nehmen wir Stripperköpfe von 3-4mm Durchmesser und verwenden gerne einen Stab-Stripper, weil hiermit das Ausleiten am distalen Insuffizienzpunkt erheblich leichter ist. Das konventionelle Stripping mit Freilegung der V. saphena magna am Knöchel und antegradem Stripping von distal nach proximal wird aufgrund des erhöhten Risikos der sensiblen Nervenverletzung von uns nur in Ausnahmefällen durchgeführt.

Die Seitenastexhärese führen wir über kleinstmögliche Hautinzisionen mittels feiner Klemme oder hierfür modifiziertes VaradyHäkchen durch. Unser Häkchen hat einen deutlich längeren Arbeitsbereich und ist insgesamt etwas stabiler als das so genannte original Varady-Häkchen. Die Phlebektomie führen wir bevorzugt in Tumeszenzanästhesie durch, gelegentlich auch in Blutleere.

\section{Rezidivvarikose}

Die Rezidivvarikose wird in der Literatur unterschiedlich definiert [5]. Wir definieren die Rezidivvarikose als einen duplexsonographischen Nachweis eines Refluxes im operierten Crossebereich mit/ohne Nachweis eines Crossestumpfes.

Gerade in den letzten Jahren finden sich mehrere Arbeiten in der phlebologischen Literatur über das Auftreten und die Häufigkeit von Rezidiven nach erfolgter Crossektomie. Neben technischen Fehlern wird auch eine so genannte Neoangiogenese als Grund für das Auftreten erneuter Crosserezidive diskutiert [6]. Unstrittig ist sicher die Tatsache, dass ein Großteil der Crosserezidive auf eine fehlerhafte Operationstechnik zurückzuführen ist.

Mumme et al. [7] konnten in einer retrospektiven Studie nachweisen, dass $68 \%$ der von ihnen nachoperierten inkompletten V. saphena-magna-Crossektomien ein technischer Fehler des Erstoperateurs die Ursache für das nachfolgende Crosserezidiv war. In einer Untersuchung an mehr als 500 Patienten in Dänemark [8] konnte gezeigt werden, dass in $40 \%$ der Fälle die Rezidivvarikose auf eine fehlerhafte Erstoperation zurückzuführen war, sofern der Ersteingriff in einer allgemeinchirurgischen, nicht auf Phlebochirurgie spezialisierten Abteilung, vorgenommen wurde. In etwa gleich hohen Prozentsätzen sahen sie Rezidive, sofern der Eingriff in einer chirurgischen Praxis durchgeführt wurde. Wurde der Eingriff hingegen von einem in der Phlebochirurgie erfahrenen Operateur vorgenommen, sah man nur in $14 \%$ der Fälle Rezidive.

Da die Frage der Häufigkeit einer Rezidivvarikose zwangsläufig mit der Sorgfältigkeit der Crossektomie korreliert ist, haben wir in einer eigenen Untersuchung zu klären versucht, wie häufig und in welcher Höhe direkt von der Crosse der V. saphena magna abgehende Seitenäste anzutreffen sind [9]. In dieser prospektiven Studie haben wir über einen Zeitraum von 3 Monaten alle V.-saphena-magna-Crossektomien intraoperativ vermessen. Es wurde die bereits beschriebene erweiterte Crossektomie durchgeführt. Bei den insgesamt 241 untersuchten Beinen war in 9 Fällen der erste von der V. saphena magna abgehende Seitenast in $1 \mathrm{~mm}$ Abstand von der V. femoralis, in 28 Fällen in $2 \mathrm{~mm}$ und in 45 Fällen in 4-5 mm von der V. femoralis entfernt (Tab. 2). Aufgrund dieser Daten, kann man feststellen, dass bei unvollständiger Freilegung der Crosse im Mündungsbereich $\leq 3 \mathrm{~mm}$ ein potenzielles Rezidivrisiko von $22 \%$, bei unvollständiger Freilegung der Crosse $\leq 5 \mathrm{~mm}$ das potenzielle Rezidivrisiko $44 \%$ beträgt (Tab. 3 ).

Tab. 2 Höhenlokalisation des 1. Seitenastes

\begin{tabular}{lrr}
\hline \multicolumn{1}{c}{ Anzahl } & \multicolumn{1}{l}{ Prozent } \\
\hline $1 \mathrm{~mm}$ & 9 & $9 / 241=3,7 \%$ \\
\hline $2 \mathrm{~mm}$ & 28 & $28 / 241=11,6 \%$ \\
$3 \mathrm{~mm}$ & 17 & $17 / 241=7,1 \%$ \\
$4-5 \mathrm{~mm}$ & 45 & $45 / 241=18,6 \%$ \\
$6-8 \mathrm{~mm}$ & 47 & $47 / 241=19,5 \%$ \\
\hline$-12 \mathrm{~mm}$ & 71 & $71 / 241=29,5 \%$ \\
$13-22 \mathrm{~mm}$ & 24 & $24 / 241=10,0 \%$ \\
\hline
\end{tabular}


Tab. 3 Potenzielles Risiko eines Crosserezidives

Bei unvollständiger Freilegung der

Crosse bis zum Mündungsbereich $\leq 3 \mathrm{~mm}$

beträgt das potenzielle Rezidivrisiko

$22 \%$

Bei unvollständiger Freilegung der

Crosse bis zum Mündungsbereich $\leq 5 \mathrm{~mm}$

beträgt das potenzielle Rezidivrisiko

$44 \%$

Die Neoangiogenese als Grund für den erneuten Crossereflux ist derzeit aus wissenschaftlicher Sicht eine reine Hypothese [10]. In der Literatur wird die Neoangiogenese unterschiedlich definiert. Demzufolge schwanken die prozentualen Angaben, in denen eine Rezidivvarikose auf ein Neovaskulat zurückgeführt wird erheblich [11]. Vielfach wird der duplexsonographische Nachweis kleiner, geschlängelt verlaufender Venen im operierten Crossebereich als Neoangiogenese gewertet, ohne dass es letzendlich bewiesen ist, dass es sich tatsächlich um neoangiogenetische Gefäße handelt. Wie bereits in unserer Untersuchung zur Höhenlokalisation der von der V. saphena magna abgehenden Seitenäste dargelegt [12], ist es durchaus denkbar, dass sich hinter diesen so genannten Neovaskulaten auch primär übersehene kleine Crosseseitenäste verbergen könnten. Weiterhin könnte es sich um direkt in die V. femoralis einmündende Seitenäste handeln. Gerade die Medialseite der V. femoralis ist bei der Rezidivcrossektomie wegen plattenartiger Verwachsungen nicht immer völlig einzusehen. Unsere eigenen Untersuchungen haben gezeigt, dass sich medialseitig in ca. $1 / 3$ aller Fälle direkt in die V. femoralis einmündende Seitenastvenen finden.

Insgesamt ist die Indikationsstellung zur operativen Behandlung einer Rezidivvarikose zurückhaltend zu stellen. Eine Rezidivoperation ist oft aufwändig und erfordert Erfahrung. Die Komplikationsrate ist im Vergleich zum Primäreingriff erhöht, die Resultate sind schlechter. In einigen Fällen ist der Einsatz von duplexgesteuerter Schaumsklerosierung anstelle einer Operation sinnvoll [2].

\section{Endoluminale Verfahren}

Die endoluminalen Verfahren insbesondere die endovenöse Lasertherapie (ELT) wird in unserer Klinik bei bestimmten Indikationen (alte Patienten, adipöse Patienten, Patienten mit minimalem Reflux) bevorzugt eingesetzt. Diese Methode ist unbestritten minimal invasiv. Sie ist rein ambulant durchführbar. Patienten haben keine oder nur sehr kurze Arbeitsunfähigkeitszeiten, und die Patientenzufriedenheit ist primär hoch. Die am häufigsten assoziierten Nebenwirkungen nach ELT sind Symptome der (sub-)klinisch ausgeprägten Thrombophlebitis, Schmerzen und in seltenen Fällen auftreten einer tiefen Venenthrombose [12].

Da für die endoluminalen Verfahren bislang nur wenige prospektiv randomisierte kontrollierte Studien vorliegen und ausreichende Langzeitergebnisse fehlen, kann eine abschließende Bewertung dieser Verfahren derzeit nicht vorgenommen werden.

\section{Sklerosierungstherapie}

Die Verödung in ihren unterschiedlichen Modifikationen wird unsererseits zur Behandlung von Seitenastvarizen von retikulären bzw. Besenreiservarizen eingesetzt. Gerade bei einer Rezidiv- varikose mit geringem Crossereflux wird bevorzugt die Schaumverödung angewandt.

Nach Studienlage sind die Ergebnisse nach duplexkontrollierter Verödung und nach Verwendung aufgeschäumter Verödungsmittel deutlich besser als nach Einsatz von konventioneller Sklerosierung. Die Verödungstherapie ist unter Beachtung von Indikationen und Kontraindikationen ein effektives, kostengünstiges und komplikationsarmes Behandlungsverfahren.

\section{Schlussfolgerung}

Gerade in den letzten Jahrzehnten hat sich die apparative phlebologische Diagnostik erheblich weiterentwickelt. Diagnostische Verfahren und therapeutische Methoden sind zunehmend enger miteinander verknüpft. Auf dem Gebiet der operativen Phlebologie wurden neue Methoden etabliert (Abb. 2). Ob dies allerdings immer ein tatsächlicher Fortschritt ist, muss kritisch hinterfragt werden und kann, da Langzeituntersuchungen bis dato fehlen, letztendlich zum jetzigen Zeitpunkt nicht definitiv beantwortet werden.

Idealerweise liegen Diagnostik, operative Planung und Ausführung in einer Hand und zwar in der des konservativ und operativ ausgebildeten operativen Dermatologen/Phlebologen.

Es sollte nicht vergessen werden, dass Venenerkrankungen auf einer genetischen Disposition beruhen. Diese können auch nach exakter Therapie erneut auftreten.

\section{Literatur}

${ }^{1}$ Klüss H, Noppeney T, Gerlach H, Braunbeck W, Ehresmann U, Fischer R, Langer C, Nüllen H, Salzmann G, Schimmelpfennig L. Leitlinie zur Diagnostik und Therapie des Krampfaderleidens. Phlebologie 2004; 33: $211-221$

${ }^{2}$ Hach W, Hach-Wunderle V. Wie lassen sich die Phlebogramme verbessern? Gefäßchirurgie 2003; 8: 55-62

${ }^{3}$ Blättler W, Gerlach H, Partsch H, Marshall M, Hertel T. Leitlinie zur Diagnostik und Therapie der tiefen Bein- und Beckenvenenthrombose. Phlebologie 2003; 32: 157-163

${ }^{4}$ Jünger M, Sippel K. Erkrankungen der Venen - Diagnostik und Therapieoptionen im Wandel. Aktuelle Dermatologie 2004; 30: 407-417

${ }^{5}$ Hach W, Hach-Wunderle V. Das theoretische Verständnis der Rezidivvarikose nach Operation. Gefäßchirurgie 1998; 3: 42 - 46

${ }^{6}$ Turton E, Scott D, Richards S, Weston M, Berridge D, Kent P, Kester R. Duplex-derived Evidence of Reflux after varicose Vein Surgery: Neoreflux or Neovascularisation? Eur J vasc endovasc Surg 1999; 17: 230-233

${ }^{7}$ Mumme A, Olbrich S, Barbera L, Stücker M. Saphenofemorales Leistenrezidiv nach Stripping der V. saphena magna: technischer Fehler oder Neovaskularisation. Phlebologie 2002; 31: $38-41$

${ }^{8}$ Kjeld T, Baekgaard N. Recurrence after surgery of varices in the region of long saphenous vein. Ugeskr Laeger 2003; 165: 3007

${ }^{9}$ Kraus U, Rafi L, Janthur E, Leissner C, Stenger D. Morphologische Studie zur Crossesituation der V. saphena magna. Phlebologie 2004: A5

${ }^{10}$ Frings N, Nelle A, Tran V, Glowacki P. Unvermeidbares Rezidiv und Neoreflux nach korrekter V. saphena magna Crossektomie: Neovaskularisation? Phlebologie 2003; 32: 96 - 100

${ }^{11} \mathrm{Rij}$ A, Jones G, Hill G. Neovascularization and recurrent varicose veins: More histologic and ultrasound evidence. J vasc Surg 2004; 2: 296 302

12 Pröbstle T. Endovenöse Lasertherapie der Varikose. Phlebologie 2003; 32: $131-137$ 